Original contribution

\title{
Fractional order vs. exponential fitting in UTE MR imaging of the patellar tendon
}

\author{
D. Papp (MSc)*,1, S.J. Breda (MD) ${ }^{1}$, E.H.G. Oei (MD,PhD), D.H.J. Poot (PhD), G. Kotek (PhD), \\ J.A. Hernandez-Tamames (PhD) \\ Department of Radiology \& Nuclear Medicine, Erasmus MC University Medical Center, Doctor Molewaterplein 40, 3015 GD Rotterdam, The Netherlands
}

\section{A R T I C L E I N F O}

\section{Keywords:}

$T_{2}^{*}$ relaxation

Fractional calculus

UTE-MRI

Patellar tendon

\begin{abstract}
A B S T R A C T
Purpose: Quantification of the $T_{2}{ }^{*}$ relaxation time constant is relevant in various magnetic resonance imaging applications. Mono- or bi-exponential models are typically used to determine these parameters. However, in case of complex, heterogeneous tissues these models could lead to inaccurate results. We compared a model, provided by the fractional-order extension of the Bloch equation with the conventional models.

Methods: Axial 3D ultra-short echo time (UTE) scans were acquired using a 3.0 T MRI and a 16-channel surface coil. After image registration, voxel-wise $T_{2}{ }^{*}$ was quantified with mono-exponential, bi-exponential and fractional-order fitting. We evaluated all three models repeatability and the bias of their derived parameters by fitting at various noise levels. To investigate the effect of the SNR for the different models, a Monte-Carlo experiment with 1000 repeats was performed for different noise levels for one subject. For a cross-sectional investigation, we used the mean fitted values of the ROIs in five volunteers.

Results: Comparing the mono-exponential and the fractional order $T_{2}{ }^{*}$ maps, the fractional order fitting method yielded enhanced contrast and an improved delineation of the different tissues. In the case of the bi-exponential method, the long $T_{2}{ }^{*}$ component map demonstrated the anatomy clearly with high contrast. Simulations showed a nonzero bias of the parameters for all three mathematical models. ROI based fitting showed that the $T_{2}{ }^{*}$ values were different depending on the applied method, and they differed most for the patellar tendon in all subjects. Conclusions: In high SNR cases, the fractional order and bi-exponential models are both performing well with low bias. However, in all observed cases, one of the bi-exponential components has high standard deviation in $T_{2}{ }^{*}$. The bi-exponential model is suitable for $T_{2}{ }^{*}$ mapping, but we recommend using the fractional order model for cases of low SNR.
\end{abstract}

\section{Introduction}

Tissues with low water content such as tendons, ligaments, menisci, or cortical bone have extremely short $T_{2}{ }^{*}$ decays [1]. The MR signal of these tissues rapidly decreases with longer echo times. Therefore, in most of the routinely used MR sequences [2-5] they exhibit very low signal intensity. With short echo times, fast spin echo (FSE) sequences provide more opportunities to visualize these tissues, but due to $T_{2}$ blurring and the lack of the efficiency of the acquisition, fine-scale structures are still not well depicted [6]. However, if the echo time (TE) is drastically reduced, the signal from these tissues can be detected as well. The TE reduction gives the opportunity to characterize the tissues and to manipulate the visibility [7]. Currently there is an increasing interest in MR pulse sequences which provide extremely short echo time, such as ultrashort TE (UTE) sequence [8], zero TE (ZTE) technique [9], single point imaging technique [10], and hybrid techniques (e.g. PETRA [11] and AWSOS [12]). In order to quantify relaxation times, proper mathematical models and post-processing algorithms are as important as the acquisition strategy. Due to relatively new developments in MRI, quantification of multiple $T_{2}$ and $T_{2}{ }^{*}$ components has become available [13]. These components can be used as markers for different pathophysiological conditions. For example $T_{2}{ }^{*}$ has been proposed as a marker for subclinical changes in menisci [15]. In general, the assumption is that two types of water exist in connective tissues, free and bound water. In the bound water compartment the molecules are assumed as bound to collagen fibers or proteoglycan molecules. In case of two-component $T_{2}{ }^{*}$ analysis, the shorter component (assumed as bound water) is usually only detectable with

\footnotetext{
* Corresponding author at: Department of Radiology \& Nuclear Medicine, Erasmus Medical Center, Postbus2040 Na-2501, Rotterdam NL 3000 CA, the Netherlands. E-mail addresses: d.papp@erasmusmc.nl (D. Papp), j.hernandeztamames@erasmusmc.nl (J.A. Hernandez-Tamames).

${ }^{1}$ Co-first author.
} 
ultrashort/zero echo times sequences [30]. The problem with the multiple component analysis is the high sensitivity to noise [14,19].

In case of complex, heterogeneous or porous tissues the simple mono-exponential or sum of exponential solution of the Bloch equation cannot perfectly describe the dynamics of the relaxation. In such complex materials we observe stretched-exponential or power law behavior [20,21,26,27]. Fractional order generalization of the Bloch equation provides an alternative mathematical model to describe the observed signal in such tissues. It offers a description of the relationship between relaxation processes and internal material structure. In this study we tested the previously introduced fractional order model for patellar tendon $T_{2}{ }^{*}$ quantification. We investigated three different models using UTE acquisitions: mono-exponential, bi-exponential and fractional order models and assessed their repeatability.

\section{Methods}

In this study we compared three different mathematical models and evaluated their repeatability and the bias of their derived parameters by fitting at various noise levels. The mathematical models of signal intensity as a function of echo time were the mono-exponential, bi-exponential and fractional order models.

\subsection{Mathematical models}

Fractional calculus defines real or complex number powers of the differentiation operator as well as of the integration operator and develops a calculus for these operators that generalizes the classical operators [22,23]. We used a generalization of Bloch equations with convolution kernels from Magin et al. [[21]]. The exact forms of these kernels are unknown, and power law kernels with fading memory have been introduced. The fractional order relaxations are the following for $T_{1}$ relaxation:

$\boldsymbol{M}_{\boldsymbol{z}}(t)=\boldsymbol{M}_{\boldsymbol{z}}(0)+\left[\boldsymbol{M}_{\mathbf{0}}-\boldsymbol{M}_{\boldsymbol{z}}(0)\right]\left[\mathbf{1}-\boldsymbol{E}_{\boldsymbol{\beta}}\left[-\left(\frac{t}{T_{1}}\right)^{\beta}\right]\right]$

where $M_{z}(t)$ is the longitudinal magnetization, $M_{0}$ is the steady state magnetization, and $E_{\beta}$ is the stretched Mittag-Leffler (M-L) function: $E_{\beta}(z)=\sum_{k=0}^{\infty} \frac{z^{k}}{\Gamma(\beta k+1)}$. Note that for $\beta=1$, the M-L function is equivalent to the simple exponential function.

The fractional order $T_{2}{ }^{*}$ relaxation model is given by:

$$
\boldsymbol{M}_{F O}(T E)=\boldsymbol{M}_{x y}(0) \boldsymbol{E}_{\alpha}\left[-\left(\frac{T E}{T_{2}}\right)^{\alpha}\right]+\boldsymbol{M}_{x y}(\infty)
$$

where $M_{x y}(0)$ is the transversal magnetization at TE $=0$, and $M_{x y}(\infty)$ is the transversal magnetization at the steady state. The $M_{x y}(0)$ is related to the proton density, and $\alpha$ can be interpreted as the memory of the spin system.

The mono-exponential $T_{2}{ }^{*}$ relaxation model is given by:

$$
\boldsymbol{M}_{m}(T E)=\boldsymbol{a}_{0} \cdot e^{-\frac{T E}{T_{2}^{*}}}+\boldsymbol{a}_{1}
$$

where $a_{0}$ is the signal intensity at TE $=0$, and $a_{1}$ is the baseline [29]. The bi-exponential $T_{2}{ }^{*}$ relaxation model is given by:

$$
M_{b}(T E)=b_{0} \cdot e^{-\frac{T E}{T_{2, s}^{*}}}+b_{1} \cdot e^{-\frac{T E}{T_{2, l}^{*}}}+b_{2}
$$

where $b_{0}$ and $b_{1}$ are the component sizes, $T_{2,}{ }^{*}$ is the short $\mathrm{T}_{2}{ }^{*}$ component, $T_{2, l}{ }^{*}$ is the long $\mathrm{T}_{2}{ }^{*}$ component, and $b_{2}$ is the baseline.

The tissue-related parameters are the corresponding $T_{2}{ }^{*}$ values. In case of the fractional order method parameter $\alpha$ is also regarded as an intrinsic parameter.

\subsection{Volunteers}

In order to compare the performance of the different methods, we have randomly chosen 5 volunteers from a clinical study on patellar tendinopathy (PT). We have considered PT suitable to evaluate the different fitting models on a disease condition [31].

The single-center prospective observational study was approved by the local institutional review board. The volunteers were consecutively recruited between January 2016 and January 2019. To be eligible for inclusion, volunteers had to be aged 18-35 years, had to perform sports involving frequent jumping or cutting maneuvers for at least 3 times per week, and have a clinical diagnosis of patellar tendinopathy which was confirmed by ultrasound.

PT is a sports-related overuse injury of the patellar tendon occurring in tendon-loading sports, such as basketball, volleyball and soccer [16]. $\mathrm{PT}$ is associated with morphologic changes in tendon microstructure, with mucoid degeneration, increased levels of hydrophilic (water-attracting) glycosaminoglycans and water content as a result $[17,18]$.

\subsection{MR acquisition}

MRI of the symptomatic knee was performed using a 3 T MR system (Discovery 750, General Electric, Boston, Massachusetts, USA) using a flexible 3.0 T 16-channel surface coil (NeoCoil, Pewaukee, Wisconsin, USA). The volunteers were scanned feet-first in supine position with the knee flexed in 30 degrees. The knee was fixed by a support base for knee stabilization (NeoCoil, Pewaukee, Wisconsin, USA). The center of the surface coil was aligned with the patellar apex. Prior to scanning high-resolution images and 3D-UTE sequences [32,33], we acquired 3D variable flip angle FSE sequences with and without fat saturation in order to provide an overview of the entire knee and to use these images as localizer to prepare the UTE-scans. Regarding 3D-UTE-MRI, a total of 16 echoes were acquired at TEs of $0.032,0.49,0.97,2.92,4.87,6.82$, 8.77, 10.72, 13.6, 12.67, 16.57, 18.52, 18.7, 20.47, 22.42, 24.37, $26.32 \mathrm{~ms}$, where TE was defined as the start of the cones readout. The 16 echoes were acquired in 4 separate multi-echo sequences containing 4 echoes in interleaved order. For each multi-echo acquisition, the same TR was used. The full MR acquisition protocol of the patellar tendinopathy study is listed in Table 1.

\subsection{MR imaging analysis}

The post-processing of the 3D-UTE images was performed with an in-house developed script using Matlab software (R2015b; TheMathWorks). All three models where fitted to all echoes of the dataset. The relaxation time per voxel was estimated using the FIT routine, and mean relaxation time and the standard deviation inside different regions of interest (ROI) were calculated. The Mittag Leffler function $\left(E_{\alpha}\right)$ uses the implementation of Garrappa [24]. ROI's were manually drawn inside tendon (patellar tendon), bone marrow (femur) and muscle (sartorius muscle), and each ROI contained approximately 50 voxels (Fig. 1). The voxel based fitting was used to compare the resulting maps from the different models by visual inspection. ROI based fitting (fit on mean over ROI) was used for quantitative measurements, and to test the effect of the signal-to-noise ratio (SNR). Additionally the mean $T_{2}{ }^{*}$ value is given for the different models. For a cross sectional investigation we used the mean fitted values of the ROIs of five volunteers.

\subsection{Effect of signal to noise ratio}

To investigate the effect of the SNR for the different models, we performed a Monte-Carlo experiment with 1000 repeats for different noise levels in case of one volunteer. To obtain Rice distributed data with lower SNR we computed the magnitude after adding zero mean complex Gaussian noise with standard deviation $\sigma$ to the original ROI 
Table 1

Imaging protocol for patellar tendon imaging.

\begin{tabular}{|c|c|c|c|c|c|c|c|}
\hline Sequence & 3D PD CUBE & 3D PD CUBE FS & 3D ME-GRE & 3D ME-UTE & 3D ME-UTE & 3D ME-UTE & 3D ME-UTE \\
\hline Matrix & $384 \times 384$ & $384 \times 384$ & $400 \times 400$ & $252 \times 252$ & $252 \times 252$ & $252 \times 252$ & $252 \times 252$ \\
\hline Scan plane & Sagittal & Sagittal & Coronal oblique & Axial oblique & Axial oblique & Axial oblique & Axial oblique \\
\hline Fat saturation & NONE & Fat & Fat Special & 2 excitations per FS & 2 excitations per FS & 2 excitations per FS & 2 excitations per FS \\
\hline FOV $(\mathrm{cm})$ & 15.0 & 15.0 & 16.0 & 15.0 & 15.0 & 15.0 & 15.0 \\
\hline Resolution $\left(\mathrm{mm}^{3}\right)$ & $0.4 \times 0.4 \times 1.0$ & $0.4 \times 0.4 \times 1.0$ & $0.4 \mathrm{~mm}^{3}$ & $0.6 \times 0.6 \times 1.5$ & $0.6 \times 0.6 \times 1.5$ & $0.6 \times 0.6 \times 1.5$ & $0.6 \times 0.6 \times 1.5$ \\
\hline $\begin{array}{l}\text { Slice thickness } \\
\text { (mm) }\end{array}$ & 1.0 & 1.0 & 0.4 & 1.5 & 1.5 & 1.5 & 1.5 \\
\hline Number of slices & 120 & 120 & 52 & 60 & 60 & 60 & 60 \\
\hline $\mathrm{TE}$ (ms) & 30.0 & 30.0 & $\begin{array}{l}3.4 / 8.5 / 13.6 / \\
18.7\end{array}$ & $\begin{array}{l}0.032 / 4.87 / 12.67 / \\
20.47\end{array}$ & $\begin{array}{l}0.49 / 6.82 / 14.62 / \\
22.42\end{array}$ & $\begin{array}{l}0.97 / 8.77 / 16.57 / \\
24.37\end{array}$ & $\begin{array}{l}2.92 / 10.72 / 18.52 / \\
26.32\end{array}$ \\
\hline Number of echoes & 1 & 1 & 4 & 4 & 4 & 4 & 4 \\
\hline $\mathrm{TR}(\mathrm{ms})$ & 1200.0 & 1200.0 & 22 & 83.4 & 83.4 & 83.4 & 83.4 \\
\hline Flip angle $\left({ }^{\circ}\right)$ & & & 7 & 17 & 17 & 17 & 17 \\
\hline $\begin{array}{l}\text { Bandwidth } \\
( \pm \mathrm{kHz})\end{array}$ & 83.33 & 83.33 & 83.33 & 125 & 125 & 125 & 125 \\
\hline NEX & 0.5 & 0.5 & 1.0 & 1.0 & 1.0 & 1.0 & 1.0 \\
\hline Scan time (mm:ss) & 03:17 & 03:18 & $05: 27$ & $13: 15$ & $13: 15$ & $13: 15$ & $13: 15$ \\
\hline
\end{tabular}

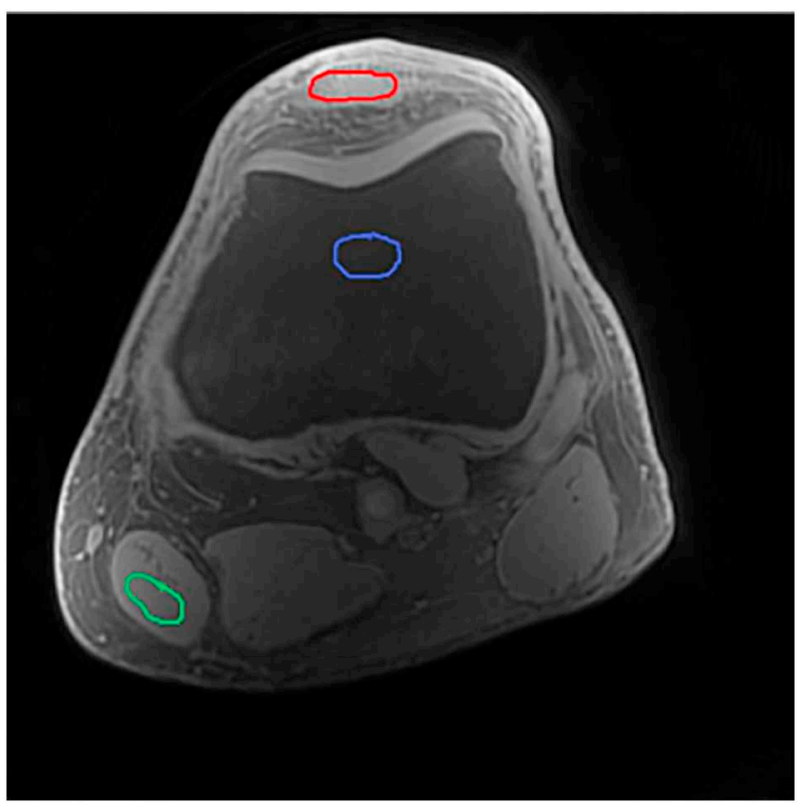

Fig. 1. ROIs for the quantitative measurements. Red - patellar tendon, blue bone marrow (femur), and green - muscle (sartorius muscle). (For interpretation of the references to color in this figure legend, the reader is referred to the web version of this article.)

means.

We selected $12 \sigma$ from $1 \%$ to $35 \%$ of the mean inside the ROI of the $\mathrm{TE}=0.032 \mathrm{~ms}$ image. All three models were fitted to the realizations. The mean and the standard deviation of the resulting parameters were taken. Two datasets were used, extracted from the ROI of the patellar tendon and the muscle. The resulting bias from the original parameters and the standard deviation were investigated. The more bias appears, the less robust is the method, and the higher the standard deviation is, the less repeatable the fitting is. The difference between the fitting results of an original derived parameter $\left(p_{o}\right)$, and the mean of that derived parameter over the 1000 Monte-Carlo simulations $\left(\overline{\boldsymbol{p}_{n}}\right)$ is what we refer to as the bias (B):

$\mathbf{B}=\frac{\left|p_{o}-\overline{p_{n}}\right|}{p_{o}} \cdot 100$.

This bias is shown as function of the noise percentage $(\mathrm{N})$, defined as:
$\mathbf{N}=\frac{1}{\mathrm{SNR}} \cdot \mathbf{1 0 0}$,

where

SNR $=\frac{\text { mean signal }(\text { inside the tissue })}{\sigma(\text { noise })}$,

where mean signal (inside the tissue at $\mathrm{TE}=0.032 \mathrm{~ms}$ ) is the mean signal value of the chosen ROI, and $\sigma$ (noise) is the standard deviation of the signal value in this ROI.

\section{Results}

The bias and the repeatability of the $T_{2}{ }^{*}$ parameter estimation using three different fitting models at different noise levels were the main interest in our investigation. Simulations showed (see in Figs. 2 and 3) a nonzero bias for all three mathematical models. Our main interest was the patellar tendon, where the original SNR was 39. For the muscle this value was 26. In case of the patellar tendon the resulting highest bias ( $\geq 200 \%$ ) appeared for only $10 \%$ of extra added noise for the short biexponential component. In contrast, the highest values for the bias for the other 2 fitting models and for the other bi-exponential component were below $80 \%$ after $35 \%$ of extra added noise. The mono-exponential and the fractional order model showed similar behavior, but the bias of the mono-exponential model's in the patellar tendon was a few percent lower. For the muscle the bias of the long bi-exponential component exceeded $1000 \%$ after $15 \%$ of extra added noise. The short bi-exponential component had a lower but still $\geq 100 \%$ bias after $20 \%$ of extra added noise. The bias of the other two models (mono-exponential and fractional order) was below $50 \%$. None of the component sizes of the bi-exponential model were negligible (Table $2 b_{s}, b_{l}$ ) so the bias was not the result of the small component size.

In the patellar tendon the $T_{2}, l^{*}$ had the highest coefficient of variation ( $\geq 50 \%$ ). The $T_{2}{ }^{*}$ and $\alpha$ values from the fractional order, the mono-exponential and the short bi-exponential models had a coefficient of variation below $15 \%$. In case of the fitting in the muscle the coefficient of variation was below $15 \%$ for all of the three models.

Fig. 4 shows representative axial $T_{2}{ }^{*}$ and $\alpha$ maps for all three models. On visual inspection, the fractional order maps showed the anatomy clearly, the contrast between tissues was higher than for the mono-exponential map, and the parameter maps were homogenous within the tissues. Clearly, when comparing the mono-exponential and the fractional order maps, the fractional order fitting method yielded enhanced contrast, an improved delineation of the different tissues, and a higher homogeneity inside a given tissue. In case of the bi-exponential method the long component map demonstrated the anatomy clearly with high contrast. Nevertheless, the short component map exhibited 


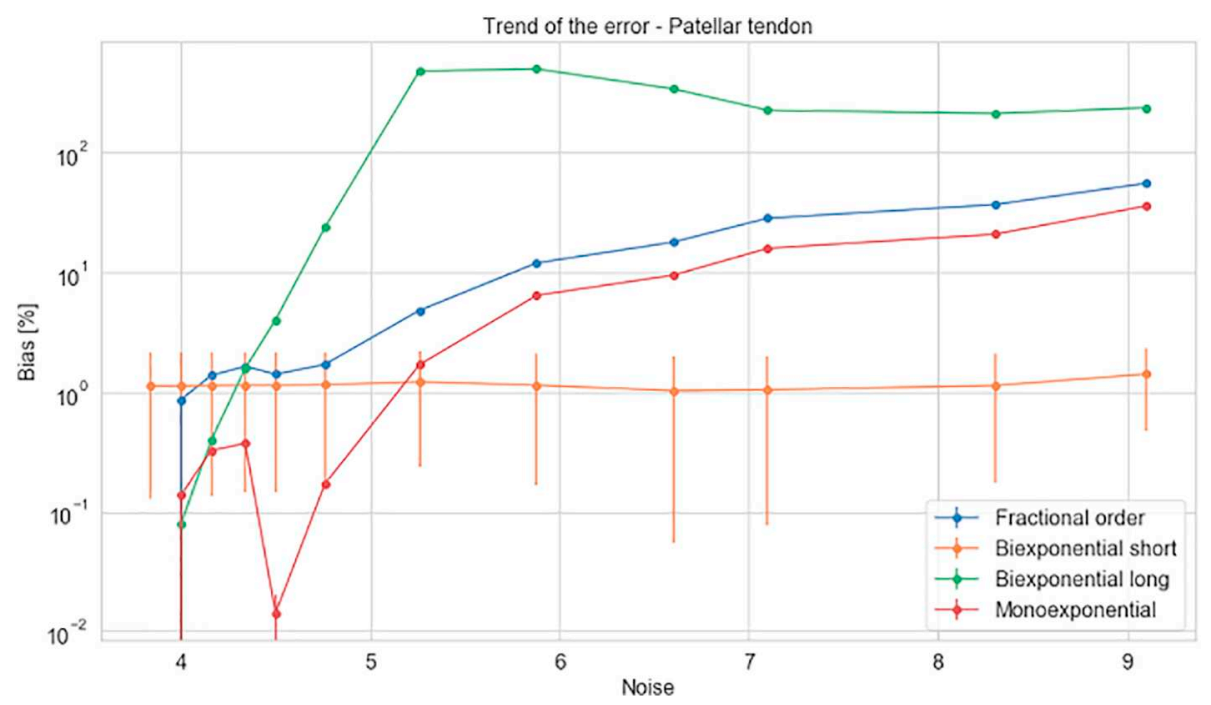

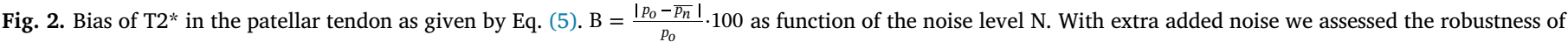

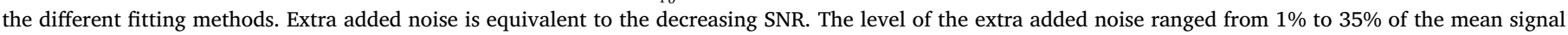

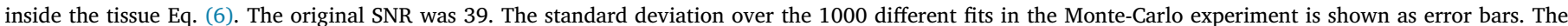
horizontal axis shows Noise $=\frac{\sigma \text { (noise) }}{\text { mean signal(inside the tissue) }} \cdot 100$ (Eq. (6).).

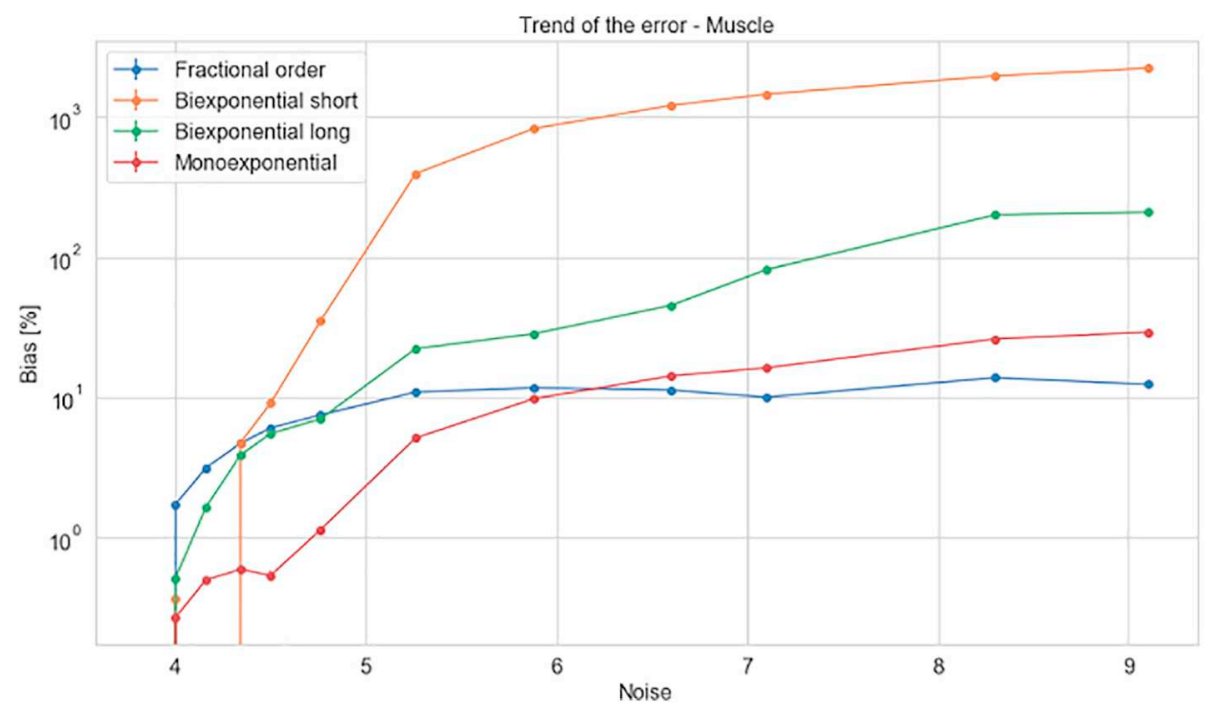

Fig. 3. Bias of $\mathrm{T} 2 *$ inside the muscle. The method is the same as for the patellar tendon. The original SNR of the muscle was 26 .

Table 2

Comparison of the different fitting methods in different tissues for one volunteer. The indicated values are the mean \pm the standard deviation of the $\boldsymbol{T}_{2}{ }^{*}$-s in [ms] and the $\alpha$ parameter. The chosen tissues were the patellar tendon, muscle (sartorius muscle) and the bone marrow (of the femur). The results are from the ROIs $\geq 50$ voxels.

\begin{tabular}{|c|c|c|c|c|c|c|c|}
\hline & \multicolumn{4}{|l|}{ Bi-exponential } & $\frac{\text { Mono-exponential }}{T_{2}^{*}[\mathrm{~ms}]}$ & \multicolumn{2}{|l|}{ Fractional order } \\
\hline Patellar tendon & $4.56 \pm 0.68$ & $7.25 \pm 9.05$ & $1412.4 \pm 247.68$ & $448.41 \pm 102.05$ & $1.4 \pm 7.41$ & $5.05 \pm 0.97$ & $0.79 \pm 0.02$ \\
\hline Bone marrow & $0.34 \pm 0.36$ & $10.31 \pm 12.79$ & $327.59 \pm 108.16$ & $112.41 \pm 105.52$ & $0.96 \pm 0.82$ & $0.51 \pm 0.15$ & $0.5 \pm 0.15$ \\
\hline
\end{tabular}

poor contrast.

Table 2 shows the mean quantitative parameters $\left(X_{i}\right.$, where i stands for the different fitting parameters) and the standard deviation over the ROI's $\left(\sigma_{i}\right)$ for one volunteer for each of the fitting methods. The $T_{2}{ }^{*}$ values were different depending on the applied method, and they differed most for the patellar tendon. In case of the muscle the derived $T_{2}{ }^{*}$ values were within the standard deviation for all of the three different models. For the bi-exponential model the component sizes were included, and this showed that none of the compartments were negligible (std $<$ mean value). The $T_{2}{ }^{*}$ values in the bi-exponential model showed the largest standard deviation among the models. In all tissues, the $T_{2}{ }^{*}$ of one of the components had a larger standard deviation than the average value. In the patellar tendon the mono-exponential model showed similar behavior to the bi-exponential one, while the $T_{2}{ }^{*}$ of the 


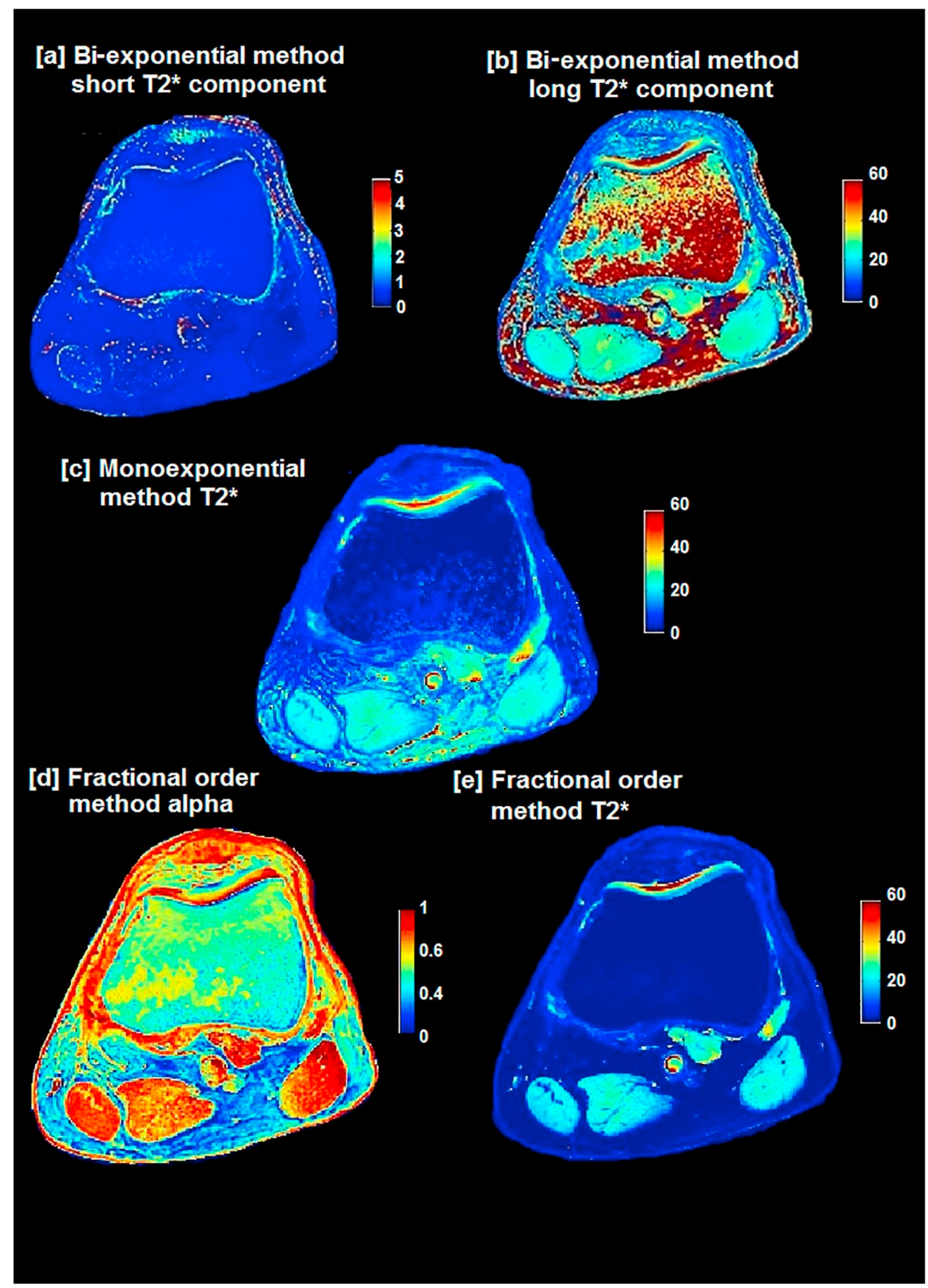

Fig. 4. [a] Bi-exponential method $T_{2, s}{ }^{*}$, [b] bi-exponential method $T_{2, l}{ }^{*}$, [c] mono-exponential method $T_{2}{ }^{*}$, [d] fractional order $\alpha$, [e] fractional order $T_{2}{ }^{*}$. $T_{2}{ }^{*}$ maps for all three different fitting models. The fitting was not performed in the background, shown black in the images. The $\alpha$ parameter is dimensionless, the $T_{2}{ }^{*}$ values are in $\mathrm{ms}$.

fractional order model had a lower standard deviation.

For ROI based fitting we made a comparison for the five subjects. Table 3 shows the mean $(\bar{X})$, and the standard deviation $\left(\sigma_{X}\right)$ of the quantitative parameters over the five volunteers and the mean of the standard deviations within the ROIs; the pooled standard deviations $(\bar{\sigma}) . \sigma_{X}$ shows the variability over their individual anatomies, $\bar{\sigma}$ contains more information by describing how much the parameters differs inside the given ROI for all volunteers. The bi-exponential model short $T_{2}{ }^{*}$ value shows the largest standard deviation for the $\bar{\sigma}$ for all three tissues. The fractional order model's highest variance is $14 \%$ for the same value, while the mono-exponential's is $15 \%$.

\section{Discussion}

Our simulations show that the results of parameter fitting vary with signal to noise ratio. One component of the bi-exponential method 
Table 3

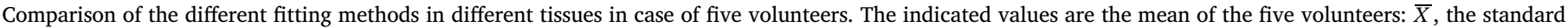

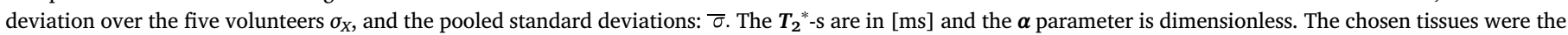
patellar tendon, muscle (sartorius muscle) and the bone marrow (of the femur). The results are from the ROIs $\geq 50$ voxels.

\begin{tabular}{|c|c|c|c|c|c|c|c|c|}
\hline & & \multicolumn{4}{|c|}{ Bi-exponential } & \multirow{2}{*}{$\begin{array}{l}\text { Mono-exponential } \\
T_{2}{ }^{*}[\mathrm{~ms}]\end{array}$} & \multicolumn{2}{|c|}{ Fractional order } \\
\hline & & $T_{2, s^{*}}[\mathrm{~ms}]$ & $T_{2,} l^{*}[\mathrm{~ms}]$ & $b_{s}$ & $b_{l}$ & & $T_{2}^{*}[\mathrm{~ms}]$ & $\alpha$ \\
\hline \multirow[t]{3}{*}{ Patellar tendon } & $\bar{X}$ & 4.01 & 15.46 & 1477.56 & 457.81 & 7.17 & 5.36 & 0.77 \\
\hline & $\sigma_{X}$ & 0.44 & 8.08 & 359.67 & 94.19 & 4.29 & 0.55 & 0.55 \\
\hline & $\bar{\sigma}$ & 4.98 & 5.18 & 52.60 & 51.59 & 0.18 & 0.46 & 0.02 \\
\hline \multirow[t]{3}{*}{ Muscle } & $\bar{X}$ & 0.49 & 9.78 & 63.19 & 332.40 & 0.62 & 0.69 & 0.46 \\
\hline & $\sigma_{X}$ & 0.02 & 3.08 & 23.30 & 31.58 & 0.06 & 0.02 & 0.04 \\
\hline & $\bar{\sigma}$ & 3.38 & 3.14 & 43.67 & 45.03 & 0.03 & 0.1 & 0.03 \\
\hline \multirow[t]{3}{*}{ Bone marrow } & $\bar{X}$ & 0.28 & 24.09 & 376.78 & 86.18 & 14.42 & 22.53 & 0.74 \\
\hline & $\sigma_{X}$ & 0.05 & 2.74 & 58.74 & 5.64 & 1.84 & 2.82 & 0.04 \\
\hline & $\bar{\sigma}$ & 0.16 & 2.63 & 24.46 & 7.20 & 0.93 & 0.71 & 0.02 \\
\hline
\end{tabular}

(depending on the type of tissue) has extremely large $(\geq 100 \%)$ bias from the original value at a given noise level after $10 \%$ of extra added noise. In the patellar tendon, the component with the larger bias is the one with the larger component value $(76 \%)$ and, in the case of the muscle, the long component ( $80 \%$ component value) has the largest bias. While the bias curve is flat, the ROI based standard deviation is the highest in the case of the bi-exponential long component, so the repeatability of the values is low. The bias curve for the mono-exponential and the fractional order models are close to each other in both the patellar tendon and the muscle. However, due to the tissue characteristics, it is known that the $T_{2}{ }^{*}$ decay curves are not simply mono-exponential. We may expect mono-exponential behavior inside the muscle, but a previous study [28] and the $\alpha$ parameter as well as the bi-exponential component sizes show that the muscle tissue is heterogeneous.

Our results clearly demonstrate that, in the case of lower SNR, the bi-exponential model has low repeatability. None of the components simultaneously has small bias and low standard deviation. In this study, the focus was on different regions of the knee, however, the acquisition was optimized for the patellar tendon, and a surface coil (NeoCoil, Pewaukee, Wisconsin, USA) was used to maximize SNR. This experimental setup resulted in sufficiently high SNR at the tendon, but that is not feasible for all the tissues. The difference in bias between the monoexponential and the fractional order model is only a few percent $(\leq 10 \%)$, and in the case of lower SNR the fractional order model has the smallest standard deviation. The heterogeneity of the tissues also indicates that the fractional order or the bi-exponential models are the most appropriate models for fitting.

The parametric maps of the different methods demonstrated differences in contrast and tissue homogeneity. Comparing the mono-exponential map to the long $T_{2}{ }^{*}$ bi-exponential map, we observed a similar trend as both of them give more contrast, and the tissue outlines are more visible. The short $T_{2}{ }^{*}$ bi-exponential map did not distinguish the different tissues clearly, and the contrast to noise level was low. The $\alpha$ map offers the best anatomical visualization along with a high tissue contrast, and it gave different values for the different tissue types. On the fractional order model $T_{2}{ }^{*}$ map, a given tissue is homogenous. The reason why we observed even a heterogeneous tissue as homogeneous (e.g. Fig. 4 fractional order $T_{2}{ }^{*}$ map, bone marrow), is because apparently the $\alpha$ value of the fractional order model captures the heterogeneity. This corresponds to the observation that, for bone marrow, the standard deviation of the fractional order model $T_{2}{ }^{*}$ is lower than that of the other two models.

Although the ROIs were chosen in the middle of the tissues - as it is shown in Fig. 1 - size and positioning could lead to some variability due to the different anatomies of the volunteers, $\sigma_{X}$ shows this difference. For the patellar tendon, the volunteers had different levels of patellar tendinopathy, which leads to higher variance as well. However $\bar{\sigma}$ shows the pooled standard deviations; the difference inside the given ROIs. We found that this reaches $>100 \%$ of $\bar{X}$ in case of the patellar tendon, and the muscle for the bi-exponential method, while in case of the other two methods it is maximum $15 \%$ of $\bar{X}$ for the $T_{2}{ }^{*}$ parameters. As $\sigma_{T 2^{*}}$ is much smaller than the difference among the $T_{2}{ }^{*}$. The main reason behind the different $T_{2}{ }^{*}$ values is the chosen fitting method as in most cases $\overline{\sigma_{T_{2}^{*}}^{*}}$ is greater than $\sigma_{T 2^{*}}$.

In conclusion, when SNR is high, a fractional order and bi-exponential model are both performing well with low bias. However, in all observed cases, one of the bi-exponential components has high standard deviation in $T_{2}{ }^{*}$ ( $\geq 50 \%$ ). The bi-exponential model is suitable for $T_{2}{ }^{*}$ mapping, but we recommend to use the fractional order model in the case of low SNR.

\section{CRediT authorship contribution statement}

D. Papp: Formal analysis, Writing - original draft. S.J. Breda: Investigation, Writing - original draft. E.H.G. Oei: Conceptualization, Writing - review \& editing. D.H.J. Poot: Formal analysis, Writing review \& editing. G. Kotek: Conceptualization, Writing - review \& editing. J.A. Hernandez-Tamames: Conceptualization, Writing - review \& editing.

\section{Acknowledgments}

The authors thank the National Basketball Association (NBA) and GE Healthcare Orthopedics and Sports Medicine Collaboration for providing the research grant and Michael Carl, PhD, Paul Baron, PhD, and Piotr Wielopolski, $\mathrm{PhD}$, for assistance in the MRI protocol optimization.

\section{Grant support}

Financial support was provided by a research grant from the National Basketball Association (NBA) and GE Healthcare Orthopedics and Sports Medicine Collaboration.

\section{References}

[1] Gatehouse PD, Bydder GM. Magnetic resonance imaging of short T2 components in tissue. Clin Radiol 2003;58:1-19.

[2] Chang Eric Y. UTE imaging in the musculoskeletal system. J Magn Reson Imaging April 2015;41(4):870-83.

[3] Gold GE, Pauly JM, Macovski A, Herfkens RJ. MR spectroscopic imaging of collagen: tendons and knee menisci. Magn Reson Med 1995;34:654-74.

[4] Robson MD, Benjamin M, Gishen P, Bydder GM. Magnetic resonance imaging of the achilles tendon using ultrashort TE (UTE) pulse sequences. Clin Radiol 2004;59:727-35.

[5] Chappell KE, Patel N, Gatehouse PD, et al. Magnetic resonance imaging of the liver with ultrashort TE (UTE) pulse sequences. J Magn Reson Imaging 2003;18:709-13.

[6] Reiter DA, Magin RL, Li W, Trujillo JJ, Pilar Velasco M, Spencer RG. Anomalous T2 relaxation in normal and degraded cartilage. Magn Reson Med 2016;76:953-62. https://doi.org/10.1002/mrm.25913. 
[7] Johnson Kevin M, et al. Optimized 3D ultrashort echo time pulmonary MRI. Magn Reson Med 2013;70(5):1241-50. [PMC. Web. 12 June 2018].

[8] Tyler DJ, Robson MD, Henkelman RM, Young IR, Bydder GM. Magnetic resonance imaging with ultrashort TE (UTE) PULSE sequences: technical considerations. J Magn Reson Imaging 2007;25:279-89. https://doi.org/10.1002/jmri.20851.

[9] Weiger M, Hennel F, Pruessmann KP. Sweep MRI with algebraic reconstruction. Magn Reson Med 2010;64:1685-95. [PubMed: 20949600].

[10] Balcom BJ, Macgregor RP, Beyea SD, Green DP, Armstrong RL, Bremner TW. Singlepoint ramped imaging with T1 enhancement (SPRITE). J Magn Reson A 1996;123:131-4. [PubMed: 8980075].

[11] Grodzki DM, Jakob PM, Heismann B. Ultrashort echo time imaging using pointwise encoding time reduction with radial acquisition (PETRA). Magn Reson Med 2012;67:510-8. [PubMed: 21721039].

[12] Qian Y, Boada FE. Acquisition-weighted stack of spirals for fast high-resolution three-dimensional ultra-short echo time MR imaging. Magn Reson Med 2008;60:135-45. [PubMed: 18581326].

[13] Juras Vladimir, et al. Bi-exponential T2* analysis of healthy and diseased Achilles tendons: an in vivo preliminary magnetic resonance study and correlation with clinical score. Eur Radiol 2013;23(10):2814-22. [PMC. Web. 12 June 2018].

[14] Barbieri S, Donati OF, Froehlich JM, Thoeny HC. Impact of the calculation algorithm on biexponential fitting of diffusion-weighted MRI in upper abdominal organs. Magn Reson Med 2016;75:2175-84. https://doi.org/10.1002/mrm.25765.

[15] Williams A, Qian Y, Golla S, Chu CR. UTE-T2 * mapping detects sub-clinical meniscus injury after anterior cruciate ligament tear. Osteoarthr Cartil/OARS Osteoarthritis Res Soc 2012;20:486-94.

[16] Zwerver J, Bredeweg SW, Van Den Akker-Scheek I. Prevalence of jumper's knee among nonelite athletes from different sports: a cross-sectional survey. Am J Sports Med 2011;39:1984-8. https://doi.org/10.1177/0363546511413370.

[17] Attia M, Scott A, Carpentier G, Lian Ø, Van Kuppevelt T, Gossard C, et al. Greater glycosaminoglycan content in human patellar tendon biopsies is associated with more pain and a lower VISA score. Br J Sports Med 2014;48:469-75. https://doi. org/10.1136/bjsports-2013-092633.

[18] Samiric T, Parkinson J, Ilic MZ, Cook J, Feller JA, Handley CJ. Changes in the composition of the extracellular matrix in patellar tendinopathy. Matrix Biol 2009;28:230-6. https://doi.org/10.1016/j.matbio.2009.04.001.

[19] Schwarcz A, Bogner P, Meric P, Correze J, Berente Z, P'al J, et al. The existence of biexponential signal decay in magnetic resonance diffusion-weighted imaging appears to be independent of compartmentalization. Magn Reson Med 2004;51:278-85. https://doi.org/10.1002/mrm.10702.

[20] Magin R, Feng X, Baleanu D. Solving the fractional order Bloch equation. Concepts Magn Reson A 2009;34:16-23.

[21] Magin RL, Li W, Pilar Velasco M, Trujillo J, Reiter DA, Morgenstern A, et al. Anomalous NMR relaxation in cartilage matrix components and native cartilage: fractional-order models. J Magn Reson 2011;210(2):184-91. https://doi.org/10. 1016/j.jmr.2011.03.006.

[22] Samko SG, Kilbas AA, Marichev OI. Fractional integrals and derivatives: theory and applications. Switzerland: Gordon and Breach; 1993.

[23] Hilfer R, editor. Applications of fractional calculus in physics. Singapore: World Scientific; 2000.

[24] Garrappa R. Numerical evaluation of two and three parameter Mittag-Leffler functions. SIAM J Numer Anal 2015;53(3):1350-69.

[26] Zhou XJ, Gao Q, Abdullah O, Magin RL. Studies of anomalous diffusion in the human brain using fractional order calculus. Magn Reson Med 2010;63:562-9. [PubMed: 20187164].

[27] Cole WC, Leblanc AD, Jhingran SG. The origin of biexponential T2 relaxation in muscle water. Magn Reson Med 1993;29:19-24. https://doi.org/10.1002/mrm. 1910290106.

[28] Damon BM, Ding Z, Anderson AW, Freyer AS, Gore JC. Validation of diffusion tensor MRI-based muscle fiber tracking. Magn Reson Med 2002;48:97-104. https:// doi.org/10.1002/mrm.10198.

[29] Juras V, Apprich S, Zbýň Š, Zak L, Deligianni X, Szomolanyi P, et al. Quantitative MRI analysis of menisci using biexponential T2* fitting with a variable echo time sequence. Magn Reson Med 2014;71:1015-23. https://doi.org/10.1002/mrm. 24760.

[30] Liu F, Kijowski R. Assessment of different fitting methods for in-vivo bi-component $\mathrm{T} 2 *$ analysis of human patellar tendon in magnetic resonance imaging. Muscles Ligaments Tendons J 2017;7(1):163-72. https://doi.org/10.11138/mltj/2017.7.1. 163. [Published 2017 May 10].

[31] Staroswiecki E, Bangerter NK, Gurney PT, Grafendorfer T, Gold GE, Hargreaves BA. In vivo sodium imaging of human patellar cartilage with a 3D cones sequence at $3 \mathrm{~T}$ and 7 T. J Magn Reson Imaging 2010;32(2):446-51.

[32] Nayak KS, et al. Proc. ISMRM. 2000. p. 509.

[33] Gold GE, et al. Proc. ISMRM. 2001. p. 244. 